\title{
Deregulation of the G1/S-phase transition is the proximal cause of mortality in old yeast mother cells
}

\author{
Gabriel E. Neurohr, ${ }^{1}$ Rachel L. Terry, ${ }^{2}$ Arzu Sandikci, ${ }^{1}$ Ke Zou, ${ }^{3} \mathrm{Hao} \mathrm{Li},{ }^{3}$ and Angelika Amon ${ }^{1,2}$ \\ ${ }^{1}$ David H. Koch Institute for Integrative Cancer Research, Howard Hughes Medical Institute, Massachusetts Institute of \\ Technology, Cambridge, Massachusetts 02139, USA; ${ }^{2}$ Department of Biology, Massachusetts Institute of Technology, Cambridge, \\ Massachusetts 02139, USA ${ }^{3}$ Department of Biochemistry and Biophysics, University of California at San Francisco, San Francisco, \\ California 94158, USA
}

Budding yeast cells produce a finite number of daughter cells before they die. Why old yeast cells stop dividing and die is unclear. We found that age-induced accumulation of the G1/S-phase inhibitor Whi5 and defects in G1/S cyclin transcription cause cell cycle delays and genomic instability that result in cell death. We further identified extrachromosomal rDNA (ribosomal DNA) circles (ERCs) to cause the G1/S cyclin expression defect in old cells. Spontaneous segregation of Whi5 and ERCs into daughter cells rejuvenates old mothers, but daughters that inherit these aging factors die rapidly. Our results identify deregulation of the G1/S-phase transition as the proximal cause of ageinduced proliferation decline and cell death in budding yeast.

[Keywords: aging; cell cycle; extrachromosomal rDNA circles; G1/S transition]

Supplemental material is available for this article.

Received January 22, 2018; revised version accepted June 14, 2018.

Aging affects most organisms and causes functional deterioration and an increased probability of death over time. Model organisms such as budding yeast have been instrumental in identifying conserved environmental and genetic modulators of aging. Budding yeast cells undergo a limited number of increasingly long cell divisions before they cease to divide and die (Mortimer and Johnston 1959; Kaeberlein 2010). Daughter cells are born with full replicative potential, whereas mother cells age. However, daughters of very old mother cells have a reduced life span and divide slower than daughters of young mothers (Egilmez and Jazwinski 1989; Kennedy et al. 1994), indicating that age can be segregated to the daughter cells. This observation has led to the proposal that aging factors accumulate in old mother cells with each division, causing functional deterioration that ultimately leads to cell cycle arrest and cell lysis.

Several aging factors have been identified in budding yeast. Alterations in mitochondrial structure and function are observed in aged yeast cells and are inherited asymmetrically between mother and daughter cells (Hughes and Gottschling 2012). Damaged mitochondria have been proposed to contribute to cellular aging by producing reactive oxygen species (ROS), which can damage both proteins and DNA (Seo et al. 2010). Old nuclear

Corresponding author: angelika@mit.edu

Article published online ahead of print. Article and publication date are online at http://www.genesdev.org/cgi/doi/10.1101/gad.312140.118. pores and protein aggregates containing oxidized proteins also accumulate in aged cells and have been implicated in aging (Aguilaniu et al. 2003; Erjavec et al. 2007; Shcheprova et al. 2008).

The first factor proposed to cause aging in budding yeast was extrachromosomal rDNA (ribosomal DNA) circles (ERCs) (Sinclair and Guarente 1997). ERCs are generated through recombination events within the repetitive rDNA locus (Kobayashi et al. 1998). The number of ERCs increases with age because they contain a replication origin and lack centromeres and hence are retained in the mother cell (Sinclair and Guarente 1997; Shcheprova et al. 2008). ERCs were identified as a source of replicative aging in budding yeast because mutations that influence the rate of ERC formation affect life span (Sinclair and Guarente 1997; Defossez et al. 1999; Shcheprova et al. 2008). For example, cells lacking the gene encoding the rDNA localized replication fork barrier protein Fob1 form ERCs at a lower rate and have an increased replicative life span (Defossez et al. 1999). How accumulation of ERCs affects replicative aging is not clear.

Here we characterize the final cell divisions leading up to cell death in budding yeast. We found that deregulation

(C) 2018 Neurohr et al. This article is distributed exclusively by Cold Spring Harbor Laboratory Press for the first six months after the full-issue publication date (see http://genesdev.cshlp.org/site/misc/terms.xhtml). After six months, it is available under a Creative Commons License (Attribution-NonCommercial 4.0 International), as described at http://creativecommons.org/licenses/by-nc/4.0/. 
of the G1/S-phase transition is a major cause of cell cycle defects observed in old cells. Age-induced accumulation of the cell cycle entry inhibitor Whi5 and defects in G1/S cyclin transcription cause cell cycle delays and genomic instability that ultimately result in cell death. We further identified ERCs as the cause of the G1/S cyclin gene expression defects in old cells. Remarkably, when excess Whi5 and ERCs are spontaneously segregated into daughter cells during mitosis, mother cells are rejuvenated, but the daughters that inherit these aging factors die rapidly. Our results identify misregulation of the G1/Sphase transition as the proximal cause of age-induced proliferation decline and cell death. Defects in G1/S control are also a characteristic of senescent mammalian cells, raising the interesting possibility that the ultimate causes of aging are conserved across eukaryotes.

\section{Results}

\section{G1 cyclin expression is deregulated in cell divisions leading up to cell death}

To determine how aging affects cell cycle progression, we performed whole life span imaging (Zhang et al. 2012) using Whi5 localization as an identifier of cell cycle stage. Whi5, the functional equivalent of the retinoblastoma $(\mathrm{Rb})$ protein in mammals, represses transcription of G1/S-phase genes and resides in the nucleus during G1 and in the cytoplasm during other stages of the cell cycle (Costanzo et al. 2004; de Bruin et al. 2004). Analysis of Whi5 localization revealed that during the last cell division, both G1 and the combined duration of S, G2, and $M$ phase were extended. Furthermore, the cell cycle stage in which cells finally arrested was variable. Out of 40 cells, 22 arrested as unbudded cells with Whi5 in the nucleus (G1). Eighteen cells lysed either shortly after Whi5 export from the nucleus or after an extended arrest in later cell cycle stages (Supplemental Fig. S1A,B).

Nuclear export of Whi5 allows for the rapid induction of genes required for cell cycle entry, DNA replication, and budding (Spellman et al. 1998). Interestingly, the time between complete Whi5 nuclear export and bud formation was extended in old cells (Supplemental Fig. S1C), pointing toward inefficient induction of the G1/S-phase gene cluster. To assess induction of G1/S-phase genes directly, we quantified expression of a destabilized form of GFP driven by the G1 cyclin CLN2 promoter (CLN2pr-GFP) (Mateus and Avery 2000). Induction of the CLN2 promoter, measured after complete export of Whi5 from the nucleus, became less efficient as cells approached death (Fig. 1A-C,E; Supplemental Fig. S2A,B; Supplemental Movie S1) and was nearly undetectable in $65 \%$ of final cell divisions (Supplemental Fig. S2C). Importantly, decreased $C L N 2$ promoter activity correlated with increased cell cycle duration: Cells with low CLN2 promoter activity spent seven times longer in G1 and took 1.9 times longer to pass through $\mathrm{S}, \mathrm{G} 2$, and $\mathrm{M}$ phase, and the period between Whi5 nuclear export and bud emergence was increased 1.3-fold (Fig. 1D; Supplemental Fig. S1D-F). We conclude that old cells experience CLN2 promoter induction defects that correlate with increased cell cycle duration. As defects in G1 cyclin gene transcription lead to cell cycle delays (Nasmyth and Dirick 1991; Koch et al. 1993; Skotheim et al. 2008), our results indicate that age-associated cell cycle delays are caused at least in part by the failure to induce G1 cyclins.

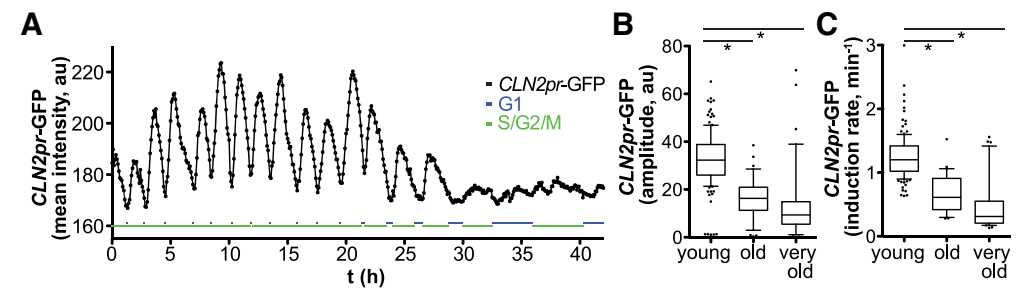

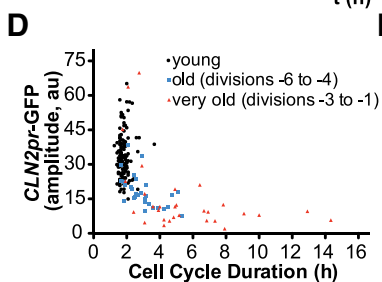

E

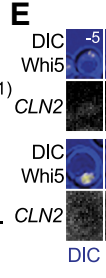

$\mathbf{F}$

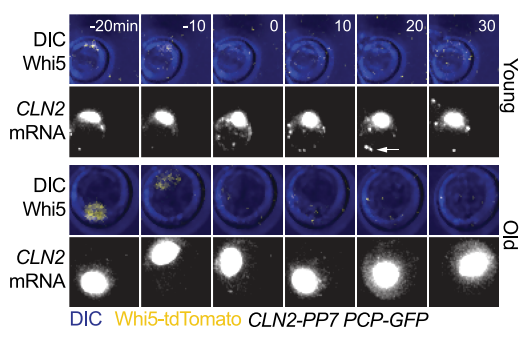

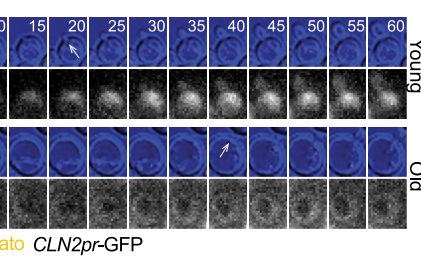

G

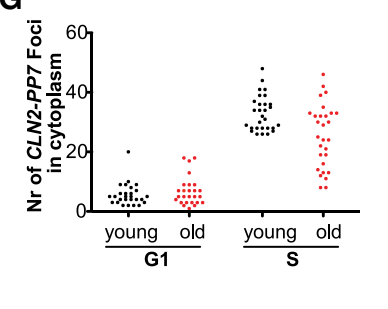

Figure 1. Age-induced cell cycle delays correlate with a $C L N 2$ expression defect. $(A-E)$ Data from whole life span imaging of cells expressing Whi5-tdTomato and CLN2pr-GFP (A36566). (A) Representative $C L N 2 p r-G F P$ activity of a single cell during its whole life span. G1 is defined as the time when Whi5 is nuclear; S/G2/M is defined by cytoplasmic Whi5. $(B, C)$ CLN2pr-GFP induction rate and amplitude determined in 10 cells during their entire life span. Young cells (all divisions except the last six cell divisions before cell death), old cells (divisions -6 to -4 before cell death), and very old cells (last three complete divisions before cell death) are compared. (*) $P<0.01$ (MannWhitney test). (D) Correlation of the CLN2pr-GFP amplitudes shown in $B$ with cell cycle duration as determined by time span between CLN2pr-GFP minima. (E) CLN2pr-GFP activation following Whi5-tdTomato nuclear export $\left(t_{0}\right)$. Arrows indicate bud emergence. $(F, G)$ Expression of CLN2-PP7 mRNA in purified old cells (A39858). (F) Image series showing CLN2-PP7 mRNA. The arrow highlights a cytoplasmic mRNA focus. The bright signal represents unbound nuclear PCP-GFP. $t_{0}=$ Whi5-tdTomato nuclear export. Cellular age as determined by bud scar labeling was as follows: for young cells, $5.5 \pm 1.5$ divisions; for old cells, $16.7 \pm 1.7$ divisions. $(G)$ Quantification of CLN2-PP7 foci before (G1) and after (S) Whi5 export. 
To determine whether the decrease in CLN2pr-GFP expression was caused by defects in CLN2 transcription, we analyzed CLN2 mRNA levels by tagging CLN2 with PP7 stem-loops (PP7). In addition, we expressed PCP-GFPNLS, which binds to the PP7 stem-loop and forms distinct foci when the mRNA is expressed (Hocine et al. 2013). Because of the bright nuclear background signal in this strain, we were not able to assess CLN2 mRNA levels in the nucleus, but old cells produced fewer cytoplasmic CLN2-PP7 foci than young cells (Fig. 1F,G; Supplemental Movie S2). Tagging CLN2 mRNA with PP7 and MS2 stem-loops (Halstead et al. 2015) led to reduced nuclear background, allowing us to analyze CLN2 mRNA levels in the nucleus. The number of CLN2-PP7-MS2 mRNA foci was also reduced in the divisions leading up to cell death (Supplemental Fig. S2D; Supplemental Movie S3). Failure to express CLN2pr-GFP in old cells is therefore caused by defects in transcription or failed stabilization of nuclear RNA.

\section{The age-associated gene expression defect affects} multiple genes

CLN2 is part of a cluster of 120 coregulated genes that are transcribed when cells pass through the G1/S transition. To test whether induction of other genes of this cluster is also impaired in aged cells, we generated fusions of a destabilized GFP with the promoters of the following genes: the G1/S cyclin CLN1, the S-phase cyclin CLB5, the gene encoding the ssDNA-binding protein Rpa (RFA1), and the gene encoding histone H2A (HTA1). Similar to the CLN2 promoter, the CLN1 and CLB5 promoters were induced less efficiently in old cells (defined as divisions -6 to -4 before cell death) and very old cells (defined as the last three complete divisions before cell death) (Fig. 2A,B; Supplemental Fig. S3A,B). In contrast, RFA1 promoter activity only mildly decreased in very old cells, and the HTA1 promoter remained fully active throughout the life span of a cell (Fig. 2C,D; Supplemental Fig. S3C,D). In agreement with previous observations, the activity amplitude of the histone promoter was increased in old cells (Supplemental Fig. S3D; Feser et al. 2010). The age-associated transcription defect therefore affects multiple, but not all, genes of the G1/S regulon. The cell cycle delays that correlate with this transcription defect are likely caused by the combined effect of multiple misregulated genes. In agreement with this interpretation, we found that overexpression of CLN2 in old cells (by replacing the endogenous CLN2 promoter with the HTA1 promoter) did not increase life span (Supplemental Fig. S4).

Are transcription defects restricted to the G1/S regulon or do old cells have a more global transcription induction problem? Analysis of expression of CLB2, which encodes a $M$-phase cyclin, revealed only minor changes in promoter activity in old cells (Fig. 2E; Supplemental Fig. S3E). In contrast, induction of MET25, a metabolically regulated gene that is activated upon removal of methionine, was impaired in old cells (defined as cells that had produced eight to 16 daughter cells) (Fig. 2F). We conclude that old cells experience transcription induction defects that
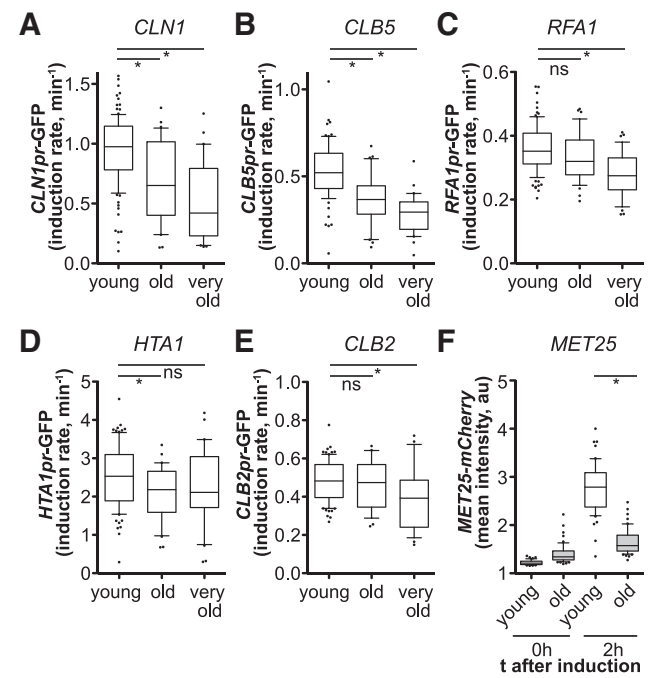

Figure 2. Widespread transcription induction defects in old cells. Whole life span imaging of cells expressing the indicated reporter constructs. $(A-E)$ Maximal GFP induction rate was determined over a sliding window of $10 \mathrm{~min}$ for each division for 10 cells per analyzed strain. For statistical analysis, the data points of young cells (all cell divisions except the last six divisions before cell death), old cells (divisions -4 to -6 before cell death), and very old cells (last three complete divisions before cell death) were combined (A40278, A40279, A40276, A40277, and A40280). (F) Cells (A39859) were grown for $2 \mathrm{~h}$ (young) or $24 \mathrm{~h}$ (old) on microfluidic chips, and MET25-mCherry was induced by switching to methionine-free medium. Only cells that had undergone a

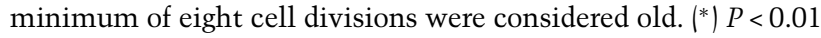
(Mann-Whitney test).

affect cell cycle-regulated and metabolically regulated genes.

\section{Aging causes DNA damage and aneuploidy}

Our data show that old cells fail to efficiently induce important cell cycle regulators at the G1/S-phase transition. Because deregulation of G1/S-phase gene transcription causes DNA replication defects (Koch et al. 1993; Schwob and Nasmyth 1993), we next determined whether S phase was affected in old cells by analyzing the localization of the ssDNA-binding protein Rpa (Rfa1-mCherry). In young cells, Rfa1-mCherry formed distinct foci in most $\mathrm{S}$ phases that were typically resolved before anaphase onset. In contrast, Rfal-mCherry foci were frequently present in old cells for $>1 \mathrm{hr}$ and often persisted into anaphase (15 out of 20 cells during their last cell division) (Fig. 3A,B; Supplemental Fig. S5A). We also analyzed the localization of Rad52, which identifies DNA double-strand breaks undergoing repair (Lisby et al. 2001). In old cells, Rad52 foci were frequently visible in nuclei for $\geq 20 \mathrm{~min}$ but were resolved prior to anaphase (Fig. 3C; Supplemental Fig. S5B, C). The rDNA locus has been described to become increasingly unstable with age and to be a hot spot for mitotic recombination in old cells (Lindstrom et al. 2011; Hu et al. 2014). Persistent Rfa1-mCherry foci fully or partially overlapped with the nucleolar marker Fob1-GFP in 12 out 
A
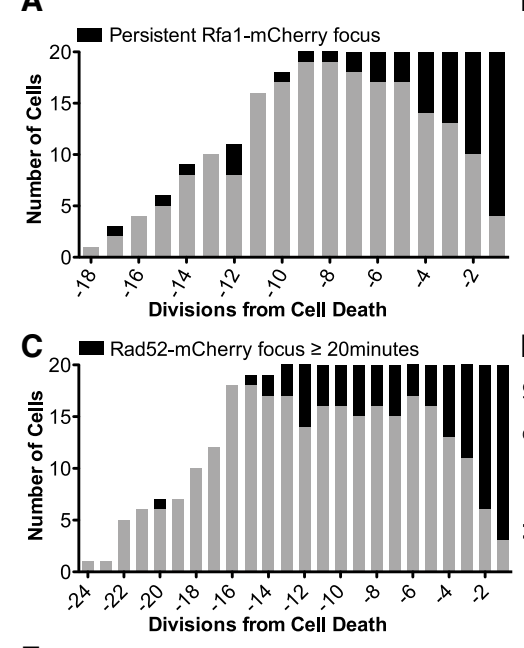

F Nuclear Fragmentation

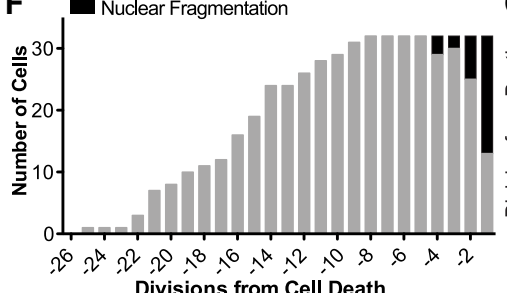

Divisions from Cell Death

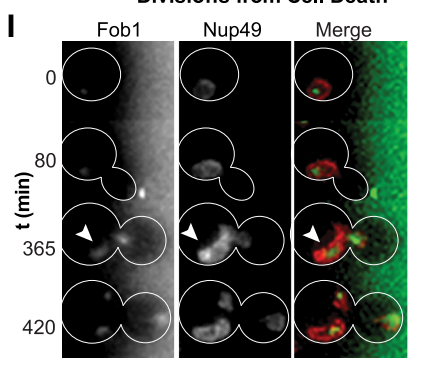

B
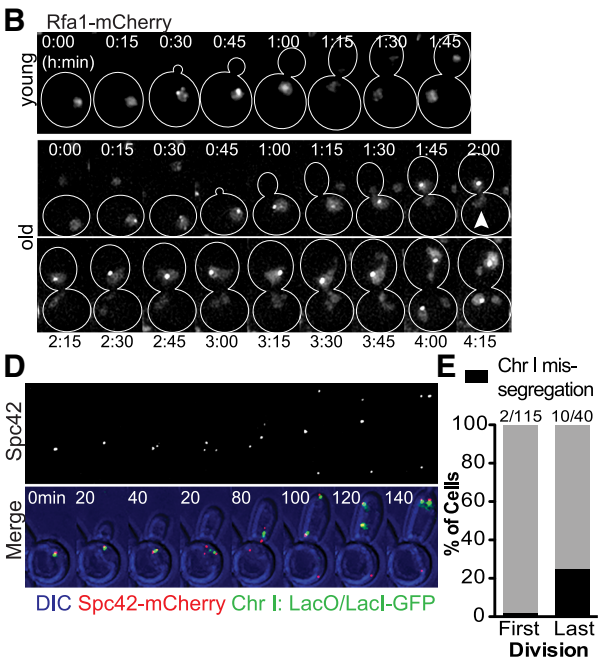

G

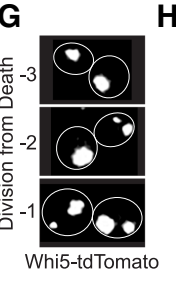

H

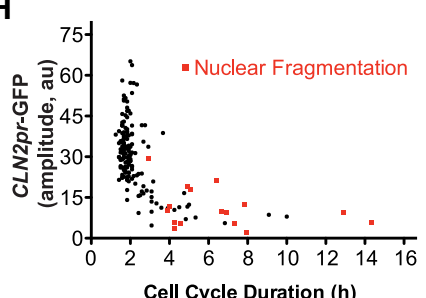

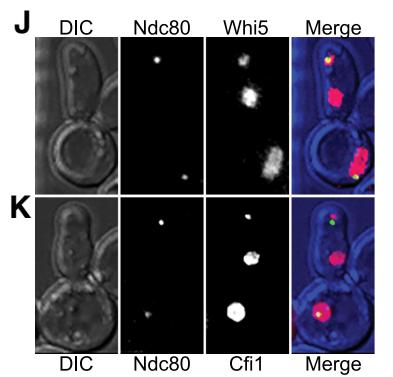

Figure 3. Persistent DNA damage and mitotic errors in aged cells. Whole life span imaging of cells expressing the indicated fluorescent proteins. $(A, B)$ Rfal-mCherry foci in divisions leading up to cell death. Rfa1-mCherry foci that did not resolve within $1 \mathrm{~h}$ after bud formation or persisted through anaphase were counted as persistent (A39864). The arrowhead in $B$ highlights a nuclear flare. $(C)$ Rad52-mCherry foci that persist for $>20 \mathrm{~min}$ in divisions leading up to cell death (A39865). (D) Formation of a tripolar spindle that results in chromosome missegregation. Spindle pole bodies were visualized using a Spc42mCherrry fusion, and chromosome I was visualized using a GFP dot $(\mathrm{LacO} / \mathrm{LacI}-$ GFP; A37624) (see the Supplemental Material). (E) Quantification of chromosome missegregation during the first and last cell divisions of a yeast cell's life. $(F-H)$ Formation of more than two nuclear compartments in telophase during the cell divisions leading up to cell death (A36566). Note that the data shown in $H$ are the same as in Figure 1D, except that nuclear fragmentations are highlighted here. (I) Nuclear fragmentation in cells expressing Nup49-mCherry and Fob1-GFP (A34453). The bright shading in the green channel is caused by autofluorescence of the medium. The arrowhead highlights a nuclear flare. $(J)$ Nuclear fragmentation in a cell expressing Ndc80-GFP and Whi5-tdTomato (A40283). (K) Nuclear fragmentation in a cell expressing Ndc80GFP and Cfil-tdTomato (A40284). of 40 cells (Supplemental Fig. S5D). We conclude that old cells experience replication defects that result in increased DNA damage and high levels of ssDNA that persist into mitosis. rDNA appears to be a site of DNA damage in old cells.

Like S-phase progression, mitosis was also severely impaired in old cells. Analysis of chromosome segregation using the LacO/LacI system to mark chromosome I with a GFP dot (see the Supplemental Material) revealed that old cells missegregated chromosomes at a high frequency. During the last division before cell death, 10 out of 40 old cells missegregated chromosome I compared with two out of 105 young cells (Fig. 3D,E). We observed two types of segregation errors. Some cells underwent a multipolar mitosis (seven out of 40 cells in last division before cell death) (Supplemental Fig. S5E), which was the cause for chromosome missegregation in three out of 10 cells. Most of these spindles (six out of seven) contained three spindle poles and formed without prior spindle pole segregation errors. This observation indicates that cells duplicate either one of their spindle poles twice or one of the spindle pole fragments. The other chromosome missegre- gation defect that we frequently observed was that the entire nucleus segregated into the bud (five out of 10 cells). Segregation of the entire nucleus into the daughter cell was usually preceded by a prolonged metaphase arrest, during which the spindle was pulled into the daughter cell. We conclude that persistent DNA damage and loss of whole chromosomes cause death in old cells.

Our live-cell analysis revealed another striking defect in old cells. Nuclei frequently fragmented during the anaphase preceding cell death (19 out of 32 cells) (Fig. 3F,G). These nuclear fragmentation events were largely restricted to cells with low CLN2 promoter activity (Fig. $3 \mathrm{H}$ ), suggesting that they could be the result of cell cycle deregulation. To characterize nuclear fragmentation further, we imaged cells that expressed the nuclear pore component Nup49 fused to mCherry. Nuclear fragmentation was always preceded by the formation of large nuclear flares (Fig. 3B,I, arrowheads point to nuclear flares). Such structures form during prolonged metaphases brought about by DNA damage or mitotic spindle defects and are known to contain rDNA (Witkin et al. 2012). Imaging of Fob1-GFP, which binds to rDNA, revealed that, indeed, 
all nuclear fragments contained rDNA (14 out of 14 nuclear fragmentation events) (Fig. 3I).

To determine whether nuclear fragmentation contributes to increased chromosome missegregation in old cells, we examined whether nuclear fragments contained chromosomes by analyzing the localization of the kinetochore component Ndc80 fused to GFP. In 31 observed nuclear fragmentation events, Ndc80-GFP localized to only two nuclear compartments (Fig. 3J,K), demonstrating that nuclear fragmentation does not contribute to the increased chromosome missegregation that occurs in old cells. The nuclear fragment lacking Ndc80 signal was typically segregated into the daughter cell (25 out of 31$)$ and contained the rDNA-binding protein Cfil (Fig. 3J,K). Old cells accumulate ERCs that lack centromeres (Sinclair and Guarente 1997). We speculate that ERCs can get separated from the main chromosome mass during prolonged metaphase arrests due to nuclear flare formation. This spatial separation could then promote fragmentation of the nucleus during anaphase, generating nuclear fragments containing ERCs but not chromosomes.

\section{Spontaneous rejuvenation of mother cells at the expense of their daughters}

Our results show that aging causes cell cycle defects, genomic instability, and nuclear fragmentation. What causes old cells to divide at a slowed pace and with decreased fidelity? Insight into this question came from the analysis of old cells that spontaneously reactivated CLN2. We observed that 13 out of 46 cells that had lost the ability to express the CLN2pr-GFP fusion due to old age spontaneously reactivated the promoter (Fig. 4A-D [M2 cell in C]; Supplemental Movie S4). Reactivation was also observed in old cells expressing the CLN2-PP7-MS2-tagged mRNA (13 out of 33 cells) (Fig. 4B; Supplemental Movie S5) and cells expressing CLN1pr-GFP and CLB5pr-GFP (data not shown). Importantly, the ability to reactivate the $C L N 2$ promoter was associated with accelerated entry and progression through the cell cycle (Fig. 4A,E,F). Here, we refer to the mitosis that precedes CLN2 reactivation and resumption of rapid cell division in old mother cells as the "rejuvenating mitosis."

Remarkably, the fates of old mother cells and their daughters were reversed when CLN2 was spontaneously reactivated in old mother cells (M2 cell in Fig. 4C). Daughter cells generated during rejuvenating mitoses (D2 cell in Fig. 4C) either did not enter the next cell cycle or did so with reduced CLN2 promoter activity and significant delay (Fig. 4G,H). Subsequent cell cycle stages were also greatly extended (Fig. 4I). Importantly, old cells that reactivated CLN2 lived longer than old cells that did not reactivate CLN2 (Fig. 4J). In contrast, D2 daughter cells produced during the rejuvenating mitosis either stopped dividing immediately or went through a few exceedingly slow cell divisions before they too stopped dividing (Fig. $4 \mathrm{~K})$. These observations demonstrate that aging factors accumulate in mother cells during their replicative life spans, where they cause CLN2 promoter inactivation and cell cycle defects. Spontaneous segregation of these
A

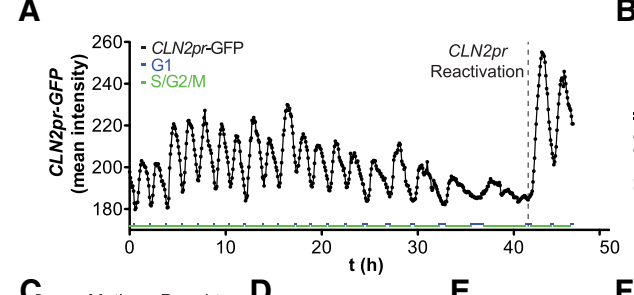

B CLN2pr Reactivation?

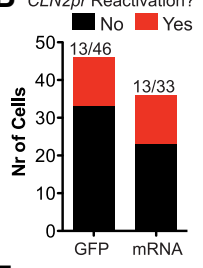

C Mother Daughter $\mathbf{D}$
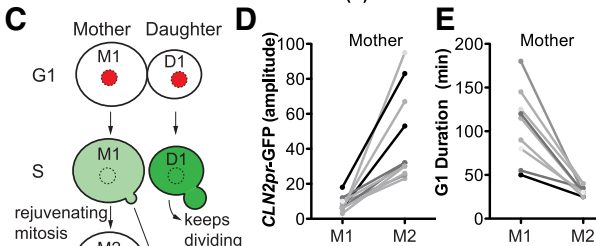

F 600 Mother
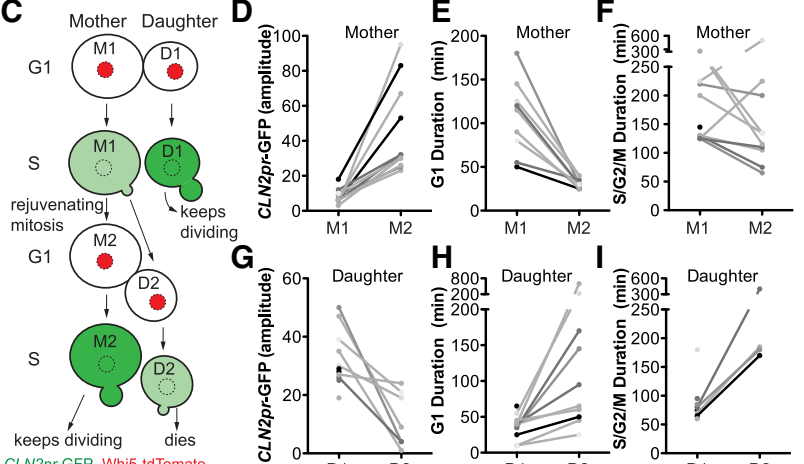

keeps dividing dies
CLN2pr-GFP Whi5-tdTomato

J
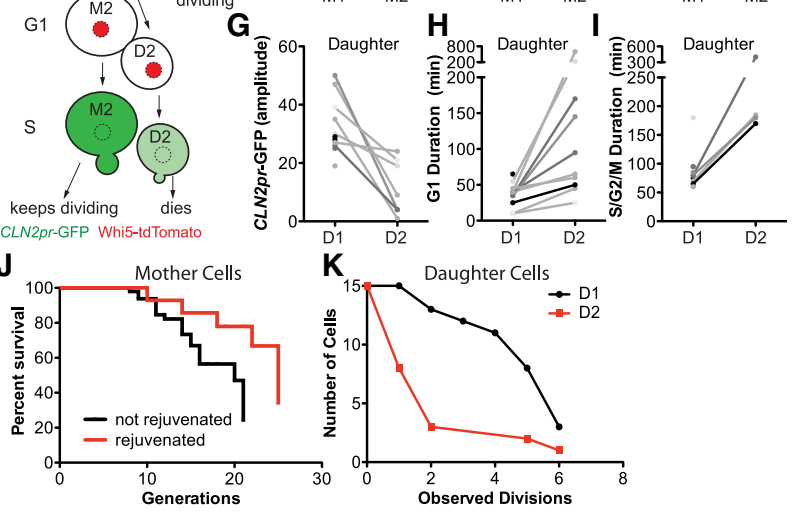

Figure 4. Spontaneous reversal of life span in old mother cells and their daughters. (A) CLN2pr-GFP activity during a yeast cell's life span. CLN2pr-GFP reactivation is indicated by a dashed line (A36566). (B) The number of cells reactivating CLN2pr-GFP (A36566) or CLN2-PP7-MS2 mRNA (A39860) (see the Supplemental Material). (C) Diagram depicting CLN2pr activity and cell fate before and after the rejuvenating mitosis. The mother cell undergoing the rejuvenating mitosis is called M1 and then M2 once the rejuvenating mitosis is completed. The daughter cell produced prior to the rejuvenating mitosis is called D1, and the daughter produced by the rejuvenating mitosis is called D2. $(D-I)$ CLN2pr-GFP amplitude and G1 (Whi5 nuclear) and S/G2/ $\mathrm{M}$ (Whi5 cytoplasmic) duration in the $\mathrm{M} 1$ and $\mathrm{M} 2$ mother $(D-F)$ and D1 and D2 daughter (G-I; A36566) cells. Points on the left side of graphs $F-I$ that are not connected by a line have no corresponding data point on the right side because the cells permanently arrested. $(J)$ The life spans of mother cells that do not undergo CLN2pr-GFP reactivation $(n=48)$ and mother cells that reactivate CLN2pr-GFP $(n=15)$. (K) The observed number of divisions of D1 and D2 daughter cells before they lyse or are washed away. $n=15$. $n$ is low because CLN2pr-GFP reactivation is a rare event.

aging factors to daughter cells rejuvenates senescent mother cells but causes rapid replicative aging in these daughters.

\section{Whi5 accumulation causes G1 delays in old cells}

What are the heritable factors that cause increased cell cycle duration in aging cells? We found that Whi5 accumulated to high levels in old cells (Fig. 5A; Supplemental 


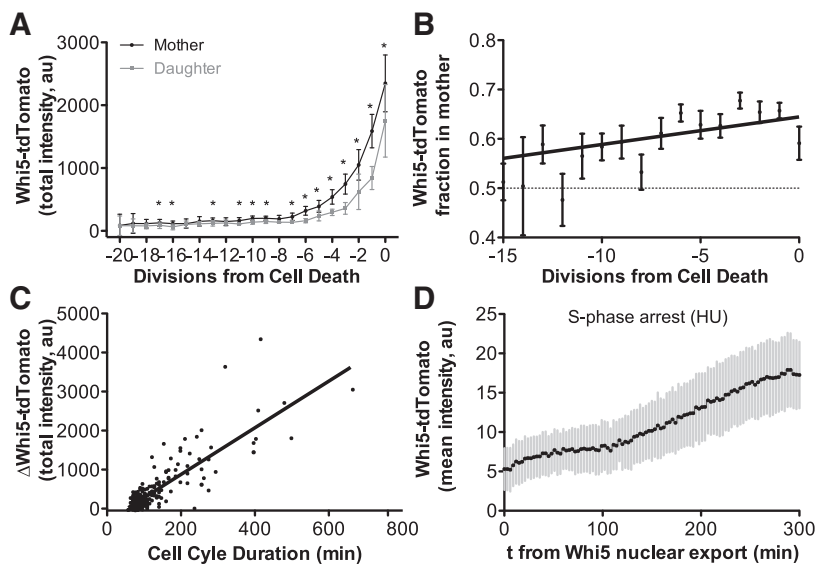

Figure 5. Whi5 accumulates in old mother cells. $(A-C)$ Whi5tdTomato signal was measured in mother and daughter cells immediately after Whi5 nuclear import during whole life span imaging. Cells that died prior to Whi5 accumulation (total Whi5 signal intensity did not reach 500; two out of 20 cells) were excluded from this analysis (A36566). $n=18$. (A) Total Whi5-tdTomato intensity (mean \pm SEM). $\left({ }^{*}\right) P<0.05$ (Wilcoxon matched pairs signed rank test). (B) Fraction of total Whi5-tdTomato partitioned into the mother cell (mean \pm SEM). (C) Increases in total Whi5-tdTomato signal between two consecutive G1 phases plotted against the corresponding cell cycle duration (budding interval). $(D)$ Whi5-tdTomato mean intensity in cells arrested in S phase with $200 \mathrm{mM}$ hydroxyurea. Measurements were started when Whi5-tdTomato nuclear export was complete (A3524; mean \pm SD). $n=202$.

Fig. S6A). This was not a consequence of increasing autofluorescence during aging (Supplemental Fig. S6B). Instead, as cells aged, Whi5 was preferentially retained in mother cells during cell division (Fig. 5A,B). In addition, Whi5-tdTomato accumulation correlated with cell cycle duration (Fig. 5C), suggesting that the Whi5 synthesis rate increases during prolonged cell cycles. Indeed, arresting young cells in early $\mathrm{S}$ phase with hydroxyurea led to an increase in Whi5 concentration (Fig. 5D). Because cell cycles become increasingly longer with age, we pro- pose that slow progression through the cell cycle drives Whi5 accumulation in old cells. Preferential retention of Whi5 in the mother cell nucleus accelerates this process. Mathematical simulation of Whi5 accumulation using the parameters determined in Figure 5, B and C, indicates that increased Whi5 synthesis during prolonged cell cycles is primarily responsible for Whi5 accumulation in old cells (Supplemental Fig. S6C; see the Supplemental Material).

Interestingly, Whi5 levels appeared to determine G1 length in old cells. Whi5 transmission into the daughter cell during rejuvenating mitoses invariably preceded CLN2 reactivation and faster cell cycles in old mother cells (Fig. 6A-E). To directly test whether high levels of Whi5 were responsible for age-induced cell cycle defects, we fused Whi5-tdTomato to an auxin-inducible degron (AID) (Nishimura et al. 2009) and expressed the fusion from the strong constitutive $A D H 1$ promoter. The fusion was functional, and addition of auxin led to rapid depletion of Whi5 (Fig. 6F; Supplemental Fig. S7A,B). Depletion of Whi5 from old cells shortened the duration of G1 but did not restore CLN2pr-GFP expression (Fig. 6G,

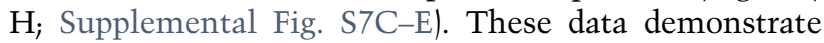
that Whi5 is an aging factor that accumulates in old cells and that Whi5 accumulation contributes to the age-associated G1 delay. Consistent with this conclusion is the observation that deletion of WHI5 extends life span (Yang et al. 2011). Our data also show that Whi5-independent mechanisms interfere with CLN2 promoter activation in old cells.

\section{ERCs interfere with G1 cyclin transcription}

We next sought to identify aging factors that contribute to the age-induced CLN2 transcription defect. ERCs accumulate in old cells and have been proposed to be aging factors (Sinclair and Guarente 1997). To determine whether ERC accumulation correlated with CLN2 promoter inactivation, we imaged cells that expressed Cfil (also known as Net1) fused to mCherry. Chromatin immunoprecipitation (ChIP) followed by sequencing (ChIP-seq) analysis of Cfil confirmed that Cfil binds exclusively to the rDNA $199 \%$
A

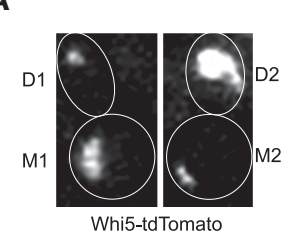

$\mathbf{F}$

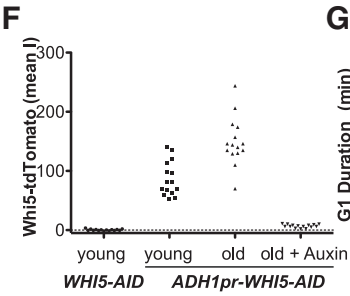

G
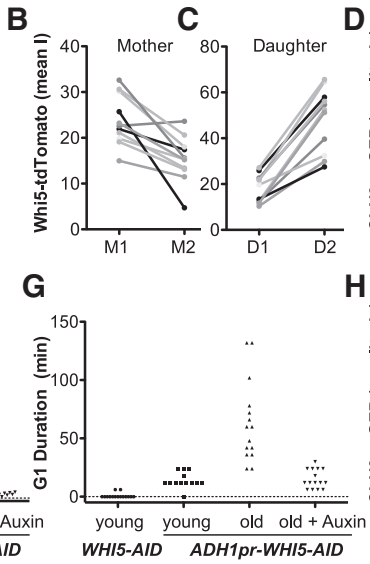

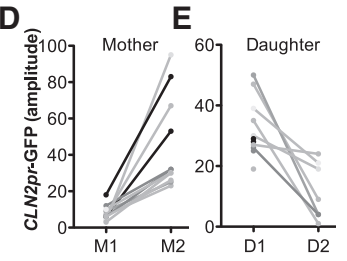

$\mathrm{H}$

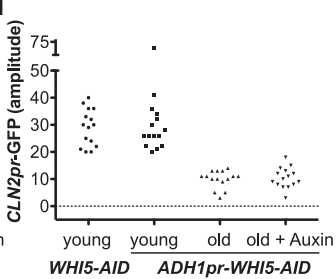

Figure 6. Accumulation of Whi5 causes a G1 delay in old cells. (A) Whi5-tdTomato (in G1) in an aging mother cell before (left) and after (right) a rejuvenating mitosis. The M1 mother cell retained most of Whi5, and the M2 mother cell has segregated Whi5 into the daughter cell. $(B-E)$ Whi5-tdTomato intensity $(B, C)$ and CLN2pr-GFP amplitude $(D, E)$ in $\mathrm{M} 1$ and $\mathrm{M} 2$ mother $(B, D)$ and D1 and D2 daughter $(C, E)$ cells (A36566). Note that the data shown in $D$ and $E$ are the same as in Figure 4, D and G, and are depicted to illustrate anti-correlation between CLN2pr-GFP amplitude and Whi5 levels. $(F-H)$ Whole life span imaging of strains expressing WHI5-tdTomato-AID or ADH1prWHI5-tdTomato-AID. Auxin was added after cells were aged. Whi5 and CLN2pr-GFP expression as well as G1 duration were analyzed in young cells (first division) and old cells before and after auxin addition (A39866 and A39867). (Mean I) Mean intensity. 
of reads allocated to a peak) and repetitive DNA adjacent to the rDNA ( $1 \%$ of reads) (X Zhou, pers. comm.). Even though we cannot exclude the possibility that Cfil binds to other DNA regions in aged cells or that it forms protein aggregates that are not bound to DNA at all, we assume that the Cfil signal correlates with the number of rDNA repeats, including ERCs. Indeed, both Cfil levels and rDNA copy number dramatically increased during aging (Fig. 7A; Supplemental Fig. S8A). Consistent with Cfil binding to ERCs, which are retained in mother cells, Cfil segregation is highly asymmetric, and the majority of the Cfil signal was retained in old mother cells during cell division (Fig. 7A, graph and left image). However, in some old mother cells, Cfil partitioned into the bud during anaphase (Fig. 7A, right image), indicating rare occasions where the majority of accumulated ERCs is inherited by daughter cells. This property of ERC inheritance allowed us to investigate whether ERC accumulation affects CLN2 expression. By simultaneously analyzing Cfi1 localization and CLN2pr-GFP expression, we observed that every old cell that reactivated the CLN2pr-GFP fusion had partitioned ERCs into the daughter cell during the rejuvenating mitosis (Fig. 7B-E; Supplemental Fig. S8B; Supplemental Movie S6).

To directly test whether accumulation of ERCs inhibits CLN2 transcription, we analyzed CLN2pr-GFP induction during aging in a mutant that accumulates fewer ERCs. fob1 $1 \Delta$ mutant cells generate ERCs at a slower rate and have an extended life span (Supplemental Fig. S8C; Defossez et al. 1999). Consistent with this extended life span, fob1 $1 \triangle$ mutants inactivated the CLN2 promoter at an older age than wild-type cells (Fig. 7F). In fact, the majority of cells died without ever inactivating the CLN2 promoter (16 out of 23 in fob1 $\Delta$ mutants vs. eight out of 27 in wild type). Importantly, ERC-mediated inhibition of CLN2 expression contributed to the cell cycle defects observed in old cells. Onset of the age-associated increase in cell cycle duration occurred later in fob $1 \Delta$ mutants than in wildtype cells (Supplemental Fig. S8D; Zhang et al. 2012). Furthermore, nuclear fragmentation and Whi5 accumulation were delayed in fob1 $1 \Delta$ mutants (Fig. 7G,H). We conclude that ERCs promote age-induced cell cycle defects and eventual cell death at least in part by interfering with transcription of CLN2 and other G1/S-phase genes.

\section{Discussion}

Many modulators of budding yeast replicative aging have been identified over the last two decades, yet an understanding of the events that lead to permanent cell cycle arrest and cell death has been missing. We identified aging factors that accumulate in mother cells during replicative aging and interfere with cell proliferation. Whi5 accumulates to high levels in old cells, leading to prolonged G1 duration. ERC accumulation interferes with the expression of G1/S-phase genes. While ERCs had been implicated in regulating life span 20 yr ago (Sinclair and Guarente 1997), this is the first demonstration of a direct link between ERC accumulation and cell cycle control.

\section{Gene expression defects accompany old age}

We found that old cells have difficulties expressing the cyclins CLN1, CLN2, and CLB5. This defect is mediated by Whi5-dependent and Whi5-independent mechanisms. Whi5 accumulates in old cells and delays activation of G1 cyclins by inhibiting the transcription factor complex Swi4/6, also known as SBF. However, even after Whi5 is exported from the nucleus, expression of CLN1 and CLN2 is delayed in old cells, indicating that even after Whi5 repression of transcription is relieved, other age-induced changes prevent full activation of G1 cyclin expression. Because all GFP reporter constructs used to examine
A
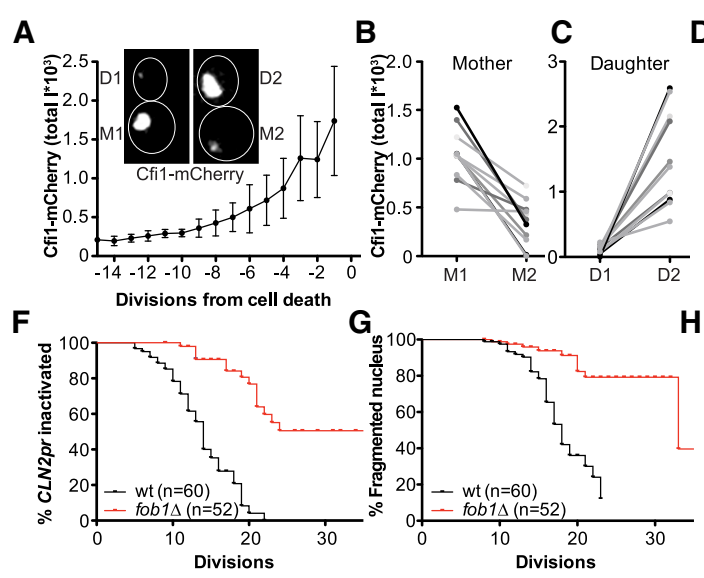

H

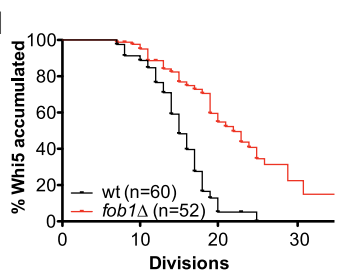

I

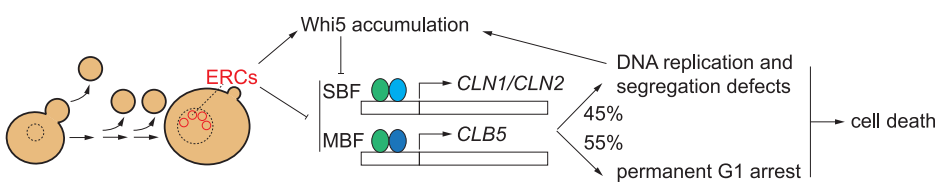

Figure 7. ERCs inhibit the G1/S-phase transition by repressing G1 cyclin expression. (A) Total CfilmCherry signal in aging mother cells (A39862). $n=$ 15. The inset shows an $\mathrm{M} 1$ mother cell that retained ERCs and an M2 mother cell that segregated ERCs into the daughter cell. $(B-E)$ Cfil-mCherry signal $(B, C)$ and $C L N 2 p r$-GFP amplitude $(D, E)$ in $M 1$ and M2 mother $(B, D)$ and D1 and D2 daughter $(C, E)$ cells (A39862). (F) First division in which CLN2pr-GFP amplitude falls below 15 is scored as CLN2 promoter inactivation in wild-type and fob1 $\Delta$ cells. This threshold corresponds to the average signal -1.5 standard deviations of values measured in young cells (A36567 and A39863). (G) Division during which the nucleus fragments for the first time in wild-type and fob1 $\Delta$ cells (A36567 and A39863). (H) Division during which mean Whi5-tdTomato intensity increases $>150$ was scored as high-level Whi 5 accumulation in wild-type and fob1s cells. The threshold corresponds to the average signal plus two standard deviations of values measured in young cells (A36567 and A39863). (I) A model for age-induced cell cycle defects. See the text for details. 
gene expression share the same GFP ORF and the same $3^{\prime}$ untranslated region and are integrated at the same locus (except for CLB5pr-GFP and CLB2pr-GFP), defects in $C L N 1, C L N 2$, and CLB5 mRNA expression must result from defective transcription initiation and/or impaired 5 ' RNA cap formation that results in reduced RNA stability. Our data indicate that the accumulation of ERCs is ultimately responsible for inhibiting CLN1, CLN2, and CLB5 expression, but the molecular mechanism by which this occurs awaits identification.

Prior reports provide evidence that expression of G1/S-phase regulon members beyond CLN1,CLN2, and $C L B 5$ is affected by old age. Levels of the DNA damage repair protein Rad51 or the cohesin subunit Mcd1/Scc1 decline with age (Pal et al. 2018). Failure to express multiple genes at the G1/S transition is expected to have severe consequences for multiple aspects of cell cycle progression, leading to pleiotropic phenotypes. Deletion of the G1 cyclins CLN1 and CLN2, for example, leads to a lengthening of G1 and stochastic cell cycle arrest (Skotheim et al. 2008). Deletion of the S-phase cyclin CLB5 slows down DNA replication (Schwob and Nasmyth 1993), and the failure to produce Rad51 and the cohesin subunit Mcd1/Scc1 impairs DNA damage repair and sister chromatid cohesion, respectively (Pal et al. 2018). These phenotypes-increased G1 duration, persistent DNA damage, and chromosome missegregation-are characteristics of old cells and correlate with impaired gene expression. The fact that old cells exhibit multiple age-associated cell cycle errors indicates that they are not caused by misregulation of an individual gene but rather are due to deregulation of multiple genes that leads to a system-wide breakdown of cell division. Consistent with this conclusion is the observation that overexpression of CLN2 from the histone HTA1 promoter does not increase life span.

The mechanisms that interfere with G1/S-phase gene expression after Whi5 export from the nucleus in old cells and how ERCs contribute to this aging phenotype remain to be determined. Global changes in histone occupancy, chromatin modifications, and transcriptional regulation have been described in senescent yeast and mammalian cells (Narita et al. 2003; Dang et al. 2009; Hu et al. 2014; Li et al. 2017). However, the fact that not all promoters are affected equally by age excludes the possibility that an essential component of the general transcription machinery becomes nonfunctional in old cells. Regulators of transcription that control expression of a subset of genes such as general transcription-associated factors (TAFs) and gene-specific transcription factors could be affected by ERC accumulation. ERCs, which can reach levels equivalent to the size of a yeast genome in old cells, could sequester TAFs, chromatin modifiers, and/or transcription factors required for G1/S gene expression. Transcription of genes of the G1/S regulon depends on either the SBF (consisting of Swi6 and Swi4) or MBF (consisting of Swi6 and Mbp1) (Wittenberg and Reed 2005). One obvious possibility is that ERCs sequester MBF and SBF because each rDNA repeat contains multiple putative MBF-binding sites (nine; ACGCGN) and SBF-binding sites (two;
CRCGAAA), and MBF was shown to bind the rDNA (Iyer et al. 2001). We tried to address this possibility by performing aging analyses in strains overexpressing SWI6, $S W I 4$, and MBP1. However, constitutive overexpression of SWI4 was toxic, and a multicopy plasmid containing all three genes was rapidly lost from cells (data not shown).

ERCs may also affect G1/S-phase gene expression indirectly; for example, by altering nuclear organization. ERCs bind to nuclear pores and cause their clustering on one side of the nucleus (Denoth-Lippuner et al. 2014). Binding of the CLN2 locus to nuclear pores represses CLN2 promoter activity (Kumar et al. 2018). ERC binding to nuclear pores could potentially stabilize this interaction. Another possibility is that high levels of RNA polymerase I (Pol I) and Pol II transcripts produced from ERCs sequester RNA processing enzymes and destabilize CLN2 and other mRNAs. However, depletion of the RNA Pol I activator Rrn9 did not lead to reactivation of the CLN2 promoter (GE Neurohr, unpubl.), arguing against this possibility. The mechanism by which ERCs interfere with G1/S-phase transcription thus awaits identification.

\section{Whi5 accumulation contributes to age-associated G1 delays}

Our results show that Whi5 accumulates in mother cells in an age-dependent manner and that high levels of the protein contribute to the long G1 periods of the cell divisions leading up to cell death. Deletion of WHI5 and other genes controlling the expression of G1/S-phase genes, such as $C L N 3$ or $B C K 2$, have been described previously to affect life span (Yang et al. 2011). Because replicative life span is inversely correlated to cell size at birth, Yang et al. (2011) concluded that the life span change observed in these mutants was caused by the altered cell size of these mutants. Our data argue for a more direct role of these mutations in regulating life span. The age-induced rise in Whi5 levels and decline in CLN1/2 promoter activity increases the likelihood of cells to permanently arrest in G1. Mutations that extend G1, such as a deletion of $C L N 3$, will synergize with the age-induced buildup of Whi5 and decrease in CLN1/2 promoter activity, leading to G1 arrest after fewer cell divisions. The result is a decreased life span.

A key question that remains to be addressed is why Whi5 accumulates in mother cells. Our data indicate that both increased Whi5 expression in old cells and asymmetric segregation of the protein contribute to this phenomenon. Whi5 expression is increased in old mother cells because as cells age, they delay in the cell cycle during which Whi5 continues to be synthesized. How Whi5 is preferentially retained in mother cells remains to be determined. It is possible that ERCs contribute to the asymmetric segregation of Whi5. This conclusion is based on the observation that during rejuvenating mitoses, the majority of both ERCs and Whi5 are segregated to the daughter cell.

There are several possibilities whereby ERCs could promote Whi5 retention in the mother cell. As mentioned earlier, each rDNA repeat contains putative SBF-binding sites (Iyer et al. 2001). Perhaps when present at extremely 
high levels, these binding sites recruit some SBF complexes and hence Whi5. Alternatively, Whi5 could be imported more efficiently into nuclei with high levels of ERCs. Nuclear pore complexes accumulate in old cells in an ERC-dependent manner (Denoth-Lippuner et al. 2014), providing a potential mechanism by which this could occur.

\section{A multipathway model for replicative aging in budding yeast}

ERC accumulation and the occurrence of age-induced gene expression defects of G1/S-phase genes are tightly linked. This perfect co-occurrence leads us to propose that ERCs interfere with expression of these genes, although we cannot exclude the possibility that ERCs also promote aging through alternative mechanisms. ERC-mediated defects in G1/S-phase gene expression are further augmented by the accumulation of Whi5 in old cells (Fig. 7I). Together, they cause substantial defects in G1/S-phase progression that leads to permanent G1 arrest in $55 \%$ of old cells. The remaining $45 \%$ of old cells progress through the cell cycle, but defects in G1/S-phase progression and mitosis cause DNA replication and segregation errors, respectively. The ensuing genome instability ultimately leads to cell death. Whether and how other aging phenotypes such as vacuolar and mitochondrial defects contribute to cell cycle lengthening and eventual cell cycle arrest and cell death remain to be determined.

While ERCs have not been found in senescing mammalian cells, a failure to induce expression of G1/S-phase genes and accumulation of cell cycle entry inhibitors are key characteristics of mammalian cell senescence (Noda et al. 1994; Narita et al. 2003). We propose that the ultimate cause of age-induced cell cycle defects and cell death-deregulation of the G1/S-phase transition-is fundamentally conserved across eukaryotes.

\section{Materials and methods}

Strains and plasmids used in this study are described in Supplemental Tables S1 and S2, respectively. All strains were derived from W303. Standard PCR-based methods were used for mRNA and protein tagging (Longtine et al. 1998; Janke et al. 2004).

Microfluidics setup, growth conditions, and fluorescence microscopy are described in detail in the Supplemental Material.

Purification of aged cells was performed as described in Unal et al. (2011). Briefly, cells were thawed from $-80^{\circ} \mathrm{C}$ stocks, plated onto YEPG $\left(2 \%\right.$ glycerol) plates, grown for $24 \mathrm{~h}$ at $30^{\circ} \mathrm{C}$, restreaked on YEPD ( $2 \%$ glucose) plates, and grown for another $24 \mathrm{~h}$. Cells were then grown in YEPD ( $2 \%$ glucose supplemented with $0.055 \mathrm{mg} / \mathrm{mL}$ adenine, $0.8 \mathrm{mg} / \mathrm{mL}$ tryptophan) containing $100 \mu \mathrm{g} / \mathrm{mL}$ ampicillin to exponential phase. Cells were harvested and labeled with $8 \mathrm{mg} / \mathrm{mL}$ biotin (Pierce Chemical) for $30 \mathrm{~min}$ at $4^{\circ} \mathrm{C}$. Biotinylated cells were grown for $6 \mathrm{~h}$ to purify young cells and for $12-14 \mathrm{~h}$ in YEPD at $25^{\circ} \mathrm{C}$ or $30^{\circ} \mathrm{C}$ to isolate middle-aged cells. Cells were harvested and incubated with anti-biotin microbeads (MACS, Miltenyi Biotechnology, 130-090-485) in $\mathrm{PBS} / 1 \% \mathrm{BSA}$ for $15 \mathrm{~min}$ at $4^{\circ} \mathrm{C}$, washed with $\mathrm{PBS} / 1 \% \mathrm{BSA}$, and applied to LS depletion magnetic columns (Miltenyi Biotechnol- ogy, 130-042-401). Isolated cells were resuspended in medium and grown for an additional six to eight divisions, after which cell sorting was repeated to isolate old cells. Depending on the experiment, cells were either fixed in $4 \%$ formaldehyde for $5 \mathrm{~min}$ or resuspended in medium to perform live-cell analysis. Old cells were labeled with fluorophore-coupled streptavidin (Thermo Fisher Scientific) and/or wheat germ agglutinin (Invitrogen) to identify old cells and determine their age by counting bud scars. Dyes were used at 1:1000 dilution, and incubations lasted for $15 \mathrm{~min}$ in medium or PBS. Purified young cells (four generations old) were used as a young cell control for the analysis of induction of CLN2-PP7 (Fig. 1F,G).

For rDNA copy number quantification, purified aged cells were collected by centrifugation, snap-frozen in liquid nitrogen, and stored at $-80^{\circ} \mathrm{C}$. For DNA purification, cells were digested in $50 \mu \mathrm{g} / \mathrm{mL}$ zymoliase (MP Biomedicals, 8320932) for $30 \mathrm{~min}$ (young) and $50 \mathrm{~min}$ (old) at $37^{\circ} \mathrm{C}$. Complete digestion was confirmed by microscopic examination. Subsequently, cells were lysed in $50 \mathrm{mM}$ EDTA $0.6 \%$ SDS. Proteinase $\mathrm{K}$ (Roche, 3115879001 ) was added to a final concentration of $0.2 \mathrm{mg} / \mathrm{mL}$, and cells were incubated for $1 \mathrm{~h}$ at $65^{\circ} \mathrm{C}$. Phenol:chloroform extraction was performed, followed by chloroform extraction. DNA was precipitated in $0.3 \mathrm{M}$ sodium acetate $(\mathrm{pH} 5.2)$ and $50 \%$ isopropanol followed by a wash in $70 \%$ ethanol. Primers for rDNA (TTCGCCTAGACGCTCTCTTC and TGGCCTTTT CATTGGATGTT) and the single-copy gene GAL1 (GCGCAAA GGAATTACCAAGA and TACCAGGCGATCTAGCAACA) were used to determine relative rDNA copy number using SYBR Green (Fisher, TAKRR081A) by quantitative PCR on a Roche LightCycler 480 II system.

\section{Acknowledgments}

We thank Pei-Hsin Hsu for help with data analysis; Xiaoxue Zhou for sharing unpublished data; Luke Berchowitz, Jan Skotheim, and Jeffrey Shao for reagents; Alar Ainla, Bashar M Hamza, and Scott Manalis for help with microfluidics; and members of the Amon laboratory for critical reading of the manuscript. G.E.N. was funded by the Swiss National Science Foundation (P2SKP3_148494 and P300PA_160996) and EMBO (ALTF 332013). This work was supported by a National Institutes of Health grant (HD085866) to A.A., who is an investigator of the Howard Hughes Medical Institute and the Paul F. Glenn Center for Biology of Aging Research at Massachusetts Institute of Technology.

Author contributions: A.A. and G.E.N. designed the experiments, drafted the manuscript, and provided funding. G.E.N. and R.L.T. acquired and analyzed the data. A.S., K.Z., and H.L. critically supported data acquisition. All authors contributed to revising the manuscript, approved the final version of the manuscript, and agreed to be accountable for the integrity of the work.

\section{References}

Aguilaniu H, Gustafsson L, Rigoulet M, Nystrom T. 2003. Asymmetric inheritance of oxidatively damaged proteins during cytokinesis. Science 299: 1751-1753.

Costanzo M, Nishikawa JL, Tang X, Millman JS, Schub O, Breitkreuz K, Dewar D, Rupes I, Andrews B, Tyers M. 2004. CDK activity antagonizes Whi5, an inhibitor of G1/S transcription in yeast. Cell 117: 899-913.

Dang W, Steffen KK, Perry R, Dorsey JA, Johnson FB, Shilatifard A, Kaeberlein M, Kennedy BK, Berger SL. 2009. Histone H4 lysine 16 acetylation regulates cellular lifespan. Nature 459: 802-807. 
de Bruin RAM, McDonald WH, Kalashnikova TI, Yates J, Wittenberg C. 2004. Cln3 activates G1-specific transcription via phosphorylation of the SBF bound repressor Whi5. Cell 117: 887-898.

Defossez PA, Prusty R, Kaeberlein M, Lin SJ, Ferrigno P, Silver PA, Keil RL, Guarente L. 1999. Elimination of replication block protein Fob1 extends the life span of yeast mother cells. Mol Cell 3: 447-455.

Denoth-Lippuner A, Krzyzanowski MK, Stober C, Barral Y. 2014. Role of SAGA in the asymmetric segregation of DNA circles during yeast ageing. eLife 3: e03790.

Egilmez NK, Jazwinski SM. 1989. Evidence for the involvement of a cytoplasmic factor in the aging of the yeast Saccharomyces cerevisiae. I Bacteriol 171: 37-42.

Erjavec N, Larsson L, Grantham J, Nystrom T. 2007. Accelerated aging and failure to segregate damaged proteins in Sir2 mutants can be suppressed by overproducing the protein aggregation-remodeling factor Hsp104p. Genes Dev 21: 2410-2421.

Feser J, Truong D, Das C, Carson JJ, Kieft J, Harkness T, Tyler JK. 2010. Elevated histone expression promotes life span extension. Mol Cell 39: 724-735.

Halstead JM, Lionnet T, Wilbertz JH, Wippich F, Ephrussi A, Singer RH, Chao JA. 2015. An RNA biosensor for imaging the first round of translation from single cells to living animals. Science 347: 1367-1671.

Hocine S, Raymond P, Zenklusen D, Chao JA, Singer RH. 2013. Single-molecule analysis of gene expression using two-color RNA labeling in live yeast. Nat Methods 10: 119-121.

Hu Z, Chen K, Xia Z, Chavez M, Pal S, Seol J-H, Chen C-C, Li W, Tyler JK. 2014. Nucleosome loss leads to global transcriptional up-regulation and genomic instability during yeast aging. Genes Dev 28: 396-408.

Hughes AL, Gottschling DE. 2012. An early age increase in vacuolar $\mathrm{pH}$ limits mitochondrial function and lifespan in yeast. Nature 492: 261-265.

Iyer VR, Horak CE, Scafe CS, Botstein D, Snyder M, Brown PO. 2001. Genomic binding sites of the yeast cell-cycle transcription factors SBF and MBF. Nature 409: 533-538.

Janke C, Magiera MM, Rathfelder N, Taxis C, Reber S, Maekawa H, Moreno-Borchart A, Doenges G, Schwob E, Schiebel E, et al. 2004. A versatile toolbox for PCR-based tagging of yeast genes: new fluorescent proteins, more markers and promoter substitution cassettes. Yeast 21: 947-962.

Kaeberlein M. 2010. Lessons on longevity from budding yeast. Nature 464: 513-519.

Kennedy BK, Austriaco NR, Guarente L. 1994. Daughter cells of Saccharomyces cerevisiae from old mothers display a reduced life span. J Cell Biol 127: 1985-1993.

Kobayashi T, Heck DJ, Nomura M, Horiuchi T. 1998. Expansion and contraction of ribosomal DNA repeats in Saccharomyces cerevisiae: requirement of replication fork blocking (Fob1) protein and the role of RNA polymerase I. Genes Dev 12: 3821-3830.

Koch C, Moll T, Neuberg M, Ahorn H, Nasmyth K. 1993. A role for the transcription factors Mbp1 and Swi4 in progression from G1 to S phase. Science 261: 1551-1557.

Kumar A, Sharma P, Gomar-Alba M, Shcheprova Z, Daulny A, Sanmartín T, Matucci I, Funaya C, Beato M, Mendoza M. 2018. Daughter-cell-specific modulation of nuclear pore complexes controls cell cycle entry during asymmetric division. Nat Cell Biol 20: 432-442.

Li Y, Jin M, O'Laughlin R, Bittihn P, Tsimring LS, Pillus L, Hasty J, Hao N. 2017. Multigenerational silencing dynamics control cell aging. Proc Natl Acad Sci 114: 11253-11258.

Lindstrom DL, Leverich CK, Henderson KA, Gottschling DE. 2011. Replicative age induces mitotic recombination in the ri- bosomal RNA gene cluster of Saccharomyces cerevisiae. PLoS Genet 7: e1002015.

Lisby M, Rothstein R, Mortensen UH. 2001. Rad52 forms DNA repair and recombination centers during $S$ phase. Proc Natl Acad Sci 98: 8276-8282.

Longtine MS, McKenzie A, Demarini DJ, Shah NG, Wach A, Brachat A, Philippsen P, Pringle JR. 1998. Additional modules for versatile and economical PCR-based gene deletion and modification in Saccharomyces cerevisiae. Yeast 14: 953-961.

Mateus C, Avery SV. 2000. Destabilized green fluorescent protein for monitoring dynamic changes in yeast gene expression with flow cytometry. Yeast 16: 1313-1323.

Mortimer RK, Johnston JR. 1959. Life span of individual yeast cells. Nature 183: 1751-1752.

Narita M, Nũnez S, Heard E, Narita M, Lin AW, Hearn SA, Spector DL, Hannon GJ, Lowe SW. 2003. Rb-mediated heterochromatin formation and silencing of E2F target genes during cellular senescence. Cell 113: 703-716.

Nasmyth K, Dirick L. 1991. The role of SWI4 and SWI6 in the activity of G1 cyclins in yeast. Cell 66: 995-1013.

Nishimura K, Fukagawa T, Takisawa H, Kakimoto T, Kanemaki M. 2009. An auxin-based degron system for the rapid depletion of proteins in nonplant cells. Nat Methods 6: 917-922.

Noda A, Ning Y, Venable SF, Pereira-Smith OM, Smith JR. 1994. Cloning of senescent cell-derived inhibitors of DNA synthesis using an expression screen. Exp Cell Res 211: 90-98.

Pal S, Postnikoff SD, Chavez M, Tyler JK. 2018. Impaired cohesion and homologous recombination during replicative aging in budding yeast. Sci $A d v$ 4: eaaq0236.

Schwob E, Nasmyth K. 1993. CLB5 and CLB6, a new pair of B cyclins involved in DNA replication in Saccharomyces cerevisiae. Genes Dev 7: 1160-1175.

Seo AY, Joseph AM, Dutta D, Hwang JCY, Aris JP, Leeuwenburgh C. 2010. New insights into the role of mitochondria in aging: mitochondrial dynamics and more. J Cell Sci 123: 2533-2542.

Shcheprova Z, Baldi S, Frei SB, Gonnet G, Barral Y. 2008. A mechanism for asymmetric segregation of age during yeast budding. Nature 454: 728-734.

Sinclair DA, Guarente L. 1997. Extrachromosomal rDNA circles a cause of aging in yeast. Cell 91: 1033-1042.

Skotheim JM, Di Talia S, Siggia ED, Cross FR. 2008. Positive feedback of G1 cyclins ensures coherent cell cycle entry. Nature 454: 291-296.

Spellman PT, Sherlock G, Zhang MQ, Iyer VR, Anders K, Eisen MB, Brown PO, Botstein D, Futcher B. 1998. Comprehensive identification of cell cycle-regulated genes of the yeast Saccharomyces cerevisiae by microarray hybridization. Mol Biol Cell 9: 3273-3297.

Unal E, Kinde B, Amon A. 2011. Gametogenesis eliminates ageinduced cellular damage and resets life span in yeast. Science 332: 1554-1557.

Witkin KL, Chong Y, Shao S, Webster MT, Lahiri S, Walters AD, Lee B, Koh JLY, Prinz WA, Andrews BJ, et al. 2012. The budding yeast nuclear envelope adjacent to the nucleolus serves as a membrane sink during mitotic delay. Curr Biol 22: 1128-1133.

Wittenberg C, Reed SI. 2005. Cell cycle-dependent transcription in yeast: promoters, transcription factors, and transcriptomes. Oncogene 24: 2746-2755.

Yang J, Dungrawala H, Hua H, Manukyan A, Abraham L, Lane W, Mead H, Wright J, Schneider BL. 2011. Cell size and growth rate are major determinants of replicative lifespan. Cell Cycle 10: 144-155.

Zhang Y, Luo C, Zou K, Xie Z, Brandman O, Ouyang Q, Li H. 2012. Single cell analysis of yeast replicative aging using a new generation of microfluidic device. PLoS One 7: e48275. 


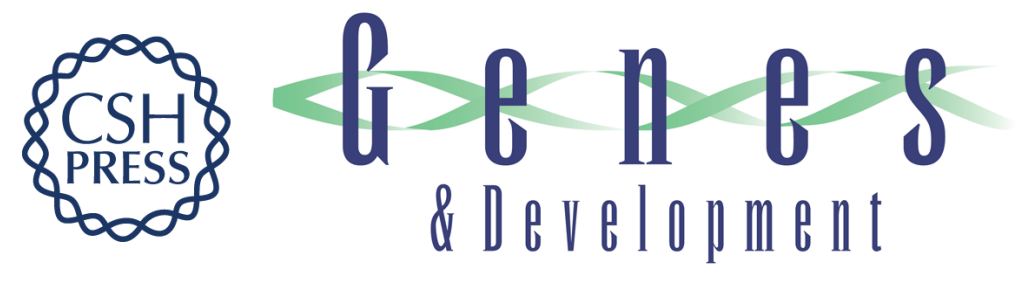

\section{Deregulation of the G1/S-phase transition is the proximal cause of mortality in old yeast mother cells}

Gabriel E. Neurohr, Rachel L. Terry, Arzu Sandikci, et al.

Genes Dev. 2018, 32: originally published online July 24, 2018

Access the most recent version at doi:10.1101/gad.312140.118

\section{Supplemental http://genesdev.cshlp.org/content/suppl/2018/07/24/gad.312140.118.DC1 Material}

References This article cites 42 articles, 15 of which can be accessed free at: http://genesdev.cshlp.org/content/32/15-16/1075.full.html\#ref-list-1

Creative This article is distributed exclusively by Cold Spring Harbor Laboratory Press for the first Commons six months after the full-issue publication date (see

License http://genesdev.cshlp.org/site/misc/terms.xhtml). After six months, it is available under a Creative Commons License (Attribution-NonCommercial 4.0 International), as described at http://creativecommons.org/licenses/by-nc/4.0/.

Email Alerting Receive free email alerts when new articles cite this article - sign up in the box at the top Service right corner of the article or click here.

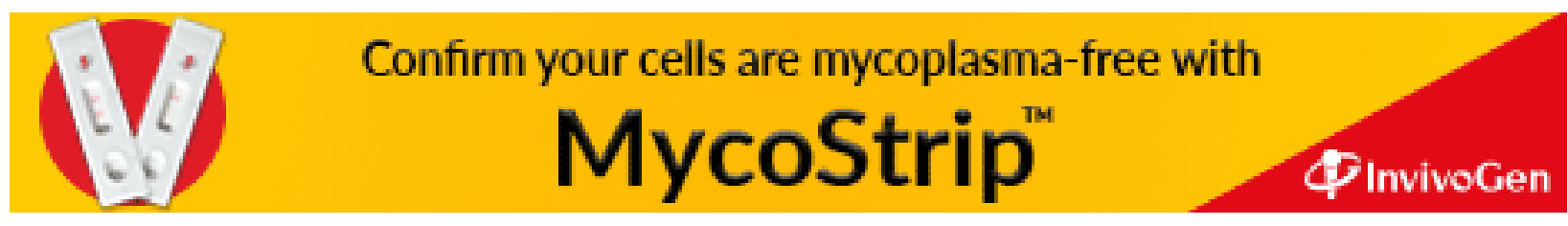

